\title{
Numerical simulation of the upward propagation of a flame in a vertical tube filled with a very lean mixture
}

\author{
F.J. Higuera* \\ E. T. S. Ingenieros Aeronáuticos, UPM, Pza. Cardenal Cisneros 3, 28040 Madrid, Spain
}

\begin{abstract}
A B S T R A C T
Upward propagation of a premixed flame in a vertical tube filled with a very lean mixture is simulated numerically using a single irreversible Arrhenius reaction model with infinitely high activation energy. In the absence of heat losses and preferential diffusion effects, a curved flame with stationary shape and velocity close to those of an open bubble ascending in the same tube is found for values of the fuel mass fraction above a certain minimum that increases with the radius of the tube, while the numerical computations cease to converge to a stationary solution below this minimum mass fraction. The vortical flow of the gas behind the flame and in its transport region is described for tubes of different radii. It is argued that this flow may become unstable when the fuel mass fraction is decreased, and that this instability, together with the flame stretch due to the strong curvature of the flame tip in narrow tubes, may be responsible for the minimum fuel mass fraction. Radiation losses and a Lewis number of the fuel slightly above unity decrease the final combustion temperature at the flame tip and increase the minimum fuel mass fraction, while a Lewis number slightly below unity has the opposite effect.
\end{abstract}

\section{Introduction}

Chemical kinetics, heat losses and nonuniformity of the gas flow affect the propagation of a flame in a gas mixture and the range of mixture composition where propagation is possible. Recognizing that the flammability limits that bound this range are not fundamental properties of the mixture but depend on the apparatus used to determine them, Coward and Jones [1] proposed to use a standard vertical tube $51 \mathrm{~mm}$ in diameter and $1.8 \mathrm{~m}$ long with an open lower end and a closed upper end. A mixture filling this tube is said to be flammable if, upon ignition at the lower end, a flame propagates all the way to the upper end of the tube; otherwise the mixture is said to be non-flammable.

Levy [2] (see also [3]) pointed out that buoyancy plays a dominant role in the upward propagation of a flame in a vertical tube near the lean flammability limit. For mixtures of methane in air, for which the Lewis number of the fuel is not far from unity, Levy [2] observed that the flame is convex toward the fresh mixture and consists of a spherical cap and a relatively long skirt, resembling an open bubble of a light fluid that rises in a tube filled with a heavy fluid. In his experiments, Levy found good agreement between the velocity of his flames and the velocity of an open bubble, as determined by Davies and Taylor [4], for tubes of the same radius; see
Shtemler and Sivashinsky [5] for a formal analysis. The effect of the radius of the tube on the measured flammability limits was investigated by Babkin et al. [6] for lean methane-air and rich propane-air mixtures. These authors find that the minimum fuel concentration for which a flame can propagate in a lean mixture increases, and the maximum fuel concentration for which it can propagate in a rich mixture decreases, when the radius of the tube increases. These results agree with previous results of Levy [2] and have been subsequently confirmed by other authors, but they contradict the results of Coward and Jones [1].

The effect of flame stretch [7] was discussed by Lewis and von Elbe [8], who concluded that this mechanism may cause the extinction of the flame observed at the flammability limits; see also Hertzberg [9]. Von Lavante and Strehlow [10] approximately computed the flow of the fresh gas above an upward propagating flame and the flame stretch, which is due mainly to the strain rate of the fresh gas flow. They showed that the stretch has a maximum at the tip of the flame, on the axis of the tube, which is the place where extinction begins at the flammability limit [2], and that the maximum stretch scaled with the inverse of the transit time of the gas across a planar flame propagating in the mixture (a dimensionless stretch or Karlovitz number) is a quantity of order unity. These results led von Lavante and Strehlow [10] to propose that flame stretch is the main cause of extinction of an upward propagating flame. Building on results derived for a planar flame in a stagnation point flow, Buckmaster and Mikolaitis [11] arrived at the conclusion that, at the flammability limit, the dimensionless 
stretch should have a certain critical value which depends on Lewis number of the fuel. Since the flame stretch decreases when the radius of the tube increases $[4,11]$, this condition predicts that the inverse of the transit time across the flame and therefore the limit fuel concentration should decrease when the radius of the tube increases. This is contrary to the experimental results of Refs. $[2,6,10]$, among others. The condition proposed in [11] relies on the assumption that convection should never bring burnt gas to the reaction region of the flame, which needs not be satisfied in a finite region around the flame tip.

Shoshin et al. [12] determined experimentally and numerically the lean flammability limit of methane-air mixtures in tubes of two different diameters. Noticing that flame stretch increases the final combustion temperature and thus strengthens the flame when the Lewis number of the fuel (Le) is smaller than unity [7], which is the case with their mixtures, these authors (see also von Lavante and Strehlow [10]) suggest that a flame propagating upwards in a tube can exist at fuel concentrations below the minimum at which a planar unstretched flame can exist. This would explain the low flammability limits obtained in narrow tubes, where the flame stretch is high. However, Shoshin and Jarosinski [13] and Shoshin et al. [14] found that the final combustion temperature of near limit methane-air flames has a minimum at the tip, which is contrary to the theoretical prediction for stretched flames with $L e<1$. This result, together with the observation that the conditions of the flow and the flame at the lean flammability limit are similar for methane-air flames with $L e<1$ and propaneair flames with $L e>1$, led these authors to consider that radiation losses from the burnt gas could be the cause of flame extinction at the flammability limit, a possibility first analyzed by Spalding [15] for a planar flame. In support of this proposal, Shoshin and coworkers note that the velocity of the burnt gas relative to the flame becomes very small in a certain region around the axis of the tube when the flammability limit is approached, which enhances radiation losses and decreases the temperature at the flame tip where extinction starts.

In this paper, the stationary upward motion of a flame in a vertical tube is simulated numerically using a single irreversible Arrhenius reaction model with infinitely high activation energy. This model cannot account for realistic kinetic effects, and in particular leaves out the thick $\mathrm{CO}$ recombination region of hydrocarbon flames, which can be easily affected by stretch, but it provides a relatively simple framework in which the buoyancy- and thermal-expansion-induced flow, and the effects of the Lewis number of the fuel and the radiation losses can be separately analyzed. The main purpose of the paper is to investigate the variation of the flow with the fuel content of the mixture and other physical parameters of the problem, and the effect of the flow on the flame. The numerical results confirm that the flow of the fresh gas and the shape and velocity of the flame are dominated by buoyancy, and also reveal a complex flow in the transport region of the flame and in the burnt gas. The numerical computations cease to converge to a stationary solution below a certain fuel concentration which increases with the radius of the tube, even in the absence of radiation losses and preferential diffusion (Le $=1$ ). While the simplified chemistry and the numerical method used are not suitable to follow the evolution of the flame down to its extinction, and therefore the numerical limit of convergence cannot be strictly identified with the experimentally determined flammability limit, the variation of this numerical limit with the radius of the tube follows the same trend as the flammability limit in Refs. $[2,6,10]$. Tentative explanations of the numerical results are given in terms of the flow- and curvature-induced flame stretch in narrow tubes and a possible instability of the vortical flow of the hot gas. Radiation losses and a $L e>1$ are found to increase the fuel concentration at the numerical limit of convergence, and a $L e<1$ decreases it.

\section{Formulation and numerical method}

\subsection{Formulation}

Consider a long cylindrical vertical tube of radius $R^{*}$, open at its lower end and closed at its upper end, which is filled with a mixture of density $\rho_{u}$, temperature $T_{u}$, and fuel mass fraction $Y_{u}$. Conditions are sought under which a flame can propagate upward along the tube with stationary shape and velocity upon ignition at the lower end of the tube. The formulation that follows is for these steadily propagating flames only. Combustion in the flame is modeled through a single irreversible Arrhenius reaction with infinitely high activation energy which fully consumes the fuel. The reaction region of the flame is then an infinitely thin free boundary where the jump conditions (9) below must be satisfied [7]. The adiabatic flame temperature is $T_{b}=T_{u}+q Y_{u} / c_{p}$, where $q$ is the heat released per unit mass of fuel consumed and $c_{p}$ is the specific heat of the mixture, which is assumed to be constant Well-known asymptotic analysis [7] shows that, in the limit $\beta=E\left(T_{b}-T_{u}\right) / \mathcal{R} T_{b}^{2} \rightarrow \infty$, where $E$ is the activation energy of the reaction and $\mathcal{R}$ is the universal gas constant, the velocity and thickness of a planar adiabatic flame propagating in the mixture are

$$
\begin{aligned}
U_{L} & =\left[2 D^{*}\left(T_{b}\right) B\right]^{1 / 2} L e \frac{T_{u} / T_{b}}{\beta} \exp \left(-\frac{E}{2 \mathcal{R} T_{b}}\right) \\
\delta_{L} & =\frac{\lambda^{*}\left(T_{u}\right)}{\rho_{u} U_{L} C_{p}},
\end{aligned}
$$

where $B$ is the frequency factor of the reaction, $D^{*}(T)$ and $\lambda^{*}(T)$ are the diffusivity of the fuel and the thermal conductivity of the gas, and $L e=\lambda^{*} /\left(\rho c_{p} D^{*}\right)$ is the Lewis number of the fuel, which is assumed to be constant.

Assuming that the reduced Lewis number $l e=\beta(L e-1)=O(1)$, scaling distances and velocities with $\delta_{L}$ and $U_{L}$, the density, viscosity $\left[\mu^{*}(T)\right]$ and thermal conductivity of the gas with $\rho_{u}, \mu^{*}\left(T_{u}\right)$ and $\lambda^{*}\left(T_{u}\right)$, and introducing the dimensionless temperature and excess of enthalpy

$\theta=\frac{T-T_{u}}{T_{b}-T_{u}} \quad$ and $\quad H=\beta\left(\theta+Y / Y_{u}-1\right)$

(where $Y$ is the fuel mass fraction scaled with $Y_{u}$ ), the equations governing the flow around a steadily propagating axisymmetric flame, are, in a reference frame moving upward with the flame,

$$
\begin{aligned}
& \boldsymbol{\nabla} \cdot(\rho \boldsymbol{v})=0, \\
& \rho \boldsymbol{v} \cdot \boldsymbol{\nabla} \boldsymbol{v}=-\nabla P+\gamma G \rho \theta \boldsymbol{i}+\operatorname{Pr} \boldsymbol{\nabla} \cdot \tau^{\prime} \\
& \rho(1+\gamma \theta)=1 \\
& \rho \boldsymbol{v} \cdot \boldsymbol{\nabla} \theta=\boldsymbol{\nabla} \cdot(\lambda \nabla \theta), \rho \boldsymbol{v} \cdot \nabla H=\nabla \cdot(\lambda \nabla H)+l e \nabla \cdot(\lambda \nabla \theta) \\
& -\Sigma\left[(1+\gamma \theta)^{4}-1\right], \\
& \mu(\theta)=\lambda(\theta)=(1+\gamma \theta)^{\kappa} .
\end{aligned}
$$

The reaction sheet is sought in the form $x=h(r)$, where $x$ and $r$ are dimensionless distances measured upward along the axis of the tube and normal to it. The jump conditions for the variables $\theta$ and $H$ at the reaction sheet are [7]

$$
\left.\begin{array}{l}
\theta=1, \quad[|H|]=0 \\
\left.\lambda(1) \frac{\partial \theta}{\partial n}\right|_{0^{-}}=\frac{\lambda(1)}{l e}\left[\left|\frac{\partial H}{\partial n}\right|\right]=e^{H / 2},
\end{array}\right\}
$$

where $[|\cdot|]=(\cdot)_{0^{+}}-(\cdot)_{0}, n$ is the distance normal to the reaction sheet, with $n>0$ in the burnt gas, and $\lambda(1)=(1+\gamma)^{\kappa}$.

Eqs. (3)-(5) are the mass and momentum conservation equations and the equation of state, written in the quasi-isobaric low Mach number approximation. Here $\tau^{\prime}=\mu\left[\nabla \boldsymbol{v}+(\nabla \boldsymbol{v})^{T}\right]$ is the non-spherical part of the viscous stress tensor scaled with $\rho_{u} U_{L}^{2}$; 
$P$ is the modified pressure $p+\rho_{u} g x$, where $g$ is the acceleration due to gravity, augmented with the spherical part of the viscous stress tensor, referred to the modified pressure of the gas around the tube and scaled with $\rho_{u} U_{L}^{2}$; and the second term on the right-hand side of $(4)$ is the dimensionless buoyancy force, with $\boldsymbol{i}$ a unit vector pointing upward. Eqs. (6) and (7) are the energy equation and the transport equation for the excess of enthalpy, which is obtained by linearly combining the conservation equations for the energy and the mass of fuel. The last term on the right-hand side of (7) is a rough model of the heat losses by radiation. Eq. (8) gives the power law temperature dependence of the dimensionless viscosity and conductivity of the gas that is adopted in what follows.

These equations are to be solved with the boundary conditions

$\boldsymbol{v}=-U_{0} \boldsymbol{i}, \quad \theta=H=0, \quad \nabla P=0 \quad$ for $\quad x \rightarrow \infty$,

where $U_{0}$ is the dimensionless velocity of the stationary flame relative to the tube, which is to be found as part of the solution by imposing the condition that the tip of the reaction sheet does not move in the reference flame tied to the flame; i.e., that $h(0)=0$ when the vertical distance is measured from the tip,

$\boldsymbol{v}=-U_{0} \boldsymbol{i}, \quad \frac{\partial \theta}{\partial r}=\frac{\partial H}{\partial r}=0 \quad$ at $\quad r=R$,

where $R=R^{*} / \delta_{L}$ is the dimensionless radius of the tube, and

$v=\theta-1=\frac{\partial H}{\partial x}=0$ for $x \rightarrow-\infty$,

where $v$ is the radial component of the velocity.

Problem (3)-(12) contains the dimensionless parameters

$\operatorname{Pr}=\frac{\mu^{*} c_{p}}{\lambda^{*}}, \quad l e=\beta(L e-1), \quad \gamma=\frac{T_{b}-T_{u}}{T_{u}}$

$G=\frac{g \delta_{L}}{U_{L}^{2}}, \quad \Sigma=\frac{\epsilon_{0} \sigma \delta_{L} \beta T_{u}^{3}}{\rho_{u} U_{L} \gamma}, \quad R=\frac{R^{*}}{\delta_{L}}, \kappa$,

which are the Prandtl number of the gas, assumed to be constant, the reduced Lewis number of the fuel, a dimensionless measure of the exothermicity of the reaction, the inverse of a Froude number, a dimensionless measure of the strength of the heat losses, where $\sigma$ is the Stefan-Boltzmann constant and $\epsilon_{0}$ is the emissivity of the species responsible for the radiation losses per unit volume of the mixture, the dimensionless radius of the tube, and the exponent in the power law (8).

The boundary condition (11) for the temperature states that the wall of the tube is adiabatic. This condition is used instead of the more common condition of a cold isothermal wall $(\theta=0)$ mostly to simplify the treatment of the problem. The reaction sheet, where $\theta=1$ according to (9), can reach an adiabatic wall but could not reach a cold wall. The infinitely thin reaction layer model would not be applicable near a cold wall, where local quenching of the reaction should be taken into account. While this is necessary to compute the flux of fuel leaking through the quenched layer and the heat transferred to the wall, such details are not thought to be important to determine the flow around the cap of the flame and its upward velocity $U_{0}$ for the large values of $R$ of interest here [1-3]. In the absence of cooling of the burnt gas downstream of the flame, the pressure of the cold gas in the upper part of a very long tube should increase linearly with time, in order to push the burnt gas over an increasing length of the tube. This, however, does not affect the governing equations above.

\subsection{Numerical method}

For the numerical treatment, the mass and momentum conservation equations (3) and (4) are written in the vorticity/stream function formulation, which is valid for stationary axisymmetric flows. The equations are rewritten in terms of the independent variables $(\xi, r)$, with $\xi=(x-\nu R) /(h(r)-v R)$ and $v$ a constant, which map the reaction sheet onto the line $\xi=1$,

The problem is discretized using second order finite differences on a rectangular grid and solved with an iterative method. For this purpose, time derivatives are added to the left-hand sides of the conservation equations (4), (6) and (7), and the equations in the second line of (9) are written as $\partial H / \partial t=\chi_{1}\left\{[|\partial H / \partial n|] / l e-. \partial \theta /\left.\partial n\right|_{0}\right\}$ and $\partial h / \partial t=\chi_{2}\left\{e^{H / 2}-\lambda(1) . \partial \theta /\left.\partial n\right|_{0}\right\}$ at $\xi=1$, where $\chi_{1}$ and $\chi_{2}$ are arbitrary constants. The first of these equations is used together with (7) to compute $H$ at the reaction sheet. The second equation is used to update the position of the reaction sheet, $h(r)$, at any $r \neq 0$. At $r=0$, where $h=0$, the equation is used with $\partial h / \partial t$ replaced by $\mathrm{d} U_{0} / \mathrm{d} t$ to update the velocity of the flame relative to the tube, $U_{0}$. The whole system is marched in time until the infinity norm of the residual becomes smaller than a tolerance typically set at $10^{-4}$.

The numerical domain extends from $\xi=0$ to $\xi=\xi_{\infty}$ and from $r=0$ to $r=R$, typically with $\xi_{\infty}=3$ and $v=1$ in the definition of $\xi$. Numerical tests showed that a nonuniform $360 \times 120$ grid leading to a spatial resolution $\Delta \xi \approx 4 \times 10^{-4}, \Delta r / R \approx 10^{-3}$ around the flame tip $(\xi=1, r=0)$ suffices to ensure grid independence of the results.

The numerical procedure is similar to that used in Ref. [16] for a related problem.

\section{Results and discussion}

\subsection{Shape and velocity of the flame}

Using the definition of $\delta_{L}$ in (1), the parameter $G$ can be rewritten as $G=\alpha_{u} g / U_{L}^{3}$, where $\alpha_{u}=\lambda^{*}\left(T_{u}\right) / \rho_{u} c_{p}$ is the thermal diffusivity of the fresh gas at its initial temperature. A base set of parameter values to be used for reference below is

$P r_{r}=1, \quad l e_{r}=0, \quad \gamma_{r}=4$,

$G_{r}=2, \quad \Sigma_{r}=0, \quad \kappa_{r}=0.75$.

Taking $\alpha_{u}=2 \times 10^{-5} \mathrm{~m}^{2} / \mathrm{s}$ for air at ambient temperature $\left(T_{u}=\right.$ $300 \mathrm{~K}$ ), the values of $\gamma_{r}$ and $G_{r}$ in (14) amount to $T_{b_{r}}=1500 \mathrm{~K}$, $U_{L_{r}}=4.63 \times 10^{-2} \mathrm{~m} / \mathrm{s}$, and $\delta_{L_{r}}=4.3 \times 10^{-4} \mathrm{~m}$ (in normal gravity), which are representative of lean methane-air mixtures slightly above the flammability limit measured in a standard flammability tube. Numerical solutions of (3)-(12) for this set of parameter values and $R=30,60$ and 90 (which amount to dimensional tube radii $R^{*}=12.9 \mathrm{~mm}, 25.8 \mathrm{~mm}$ and $38.7 \mathrm{~mm}$ ) give dimensionless flame velocities $U_{0}=3.31,4.78$ and 5.88 . These values are close to the values of the velocity of the interface between two fluids with a density ratio $\gamma+1$ rising steadily in tubes of the same radii, which are $U_{D T}=3.24,4.58$ and 5.60 according to the expression

$U_{D T}=0.467\left(\frac{\gamma}{\gamma+1} G R\right)^{1 / 2}$

derived by Davies and Taylor [4] (written here in dimensionless form for a finite density ratio). The similarity between the flame and bubble velocities reflects the dominating effect of buoyancy in both cases, which has been noted often $[2,3,10,11]$. The comparison presented here serves as a test of the numerical method used to solve (3)-(12).

Figure 1 shows some streamlines and isotherms around the tip of the flame for each of the three cases computed. (The computational domain extends some distance below the lower boundary of the figure.) As was to be expected, the flow of the fresh gas ahead of the flame resembles the flow of the heavy fluid above a bubble, with the flame playing the part of the interface between heavy and light fluids. The difference between the two flows is in the mass flux crossing the flame, which has no analogue for a 

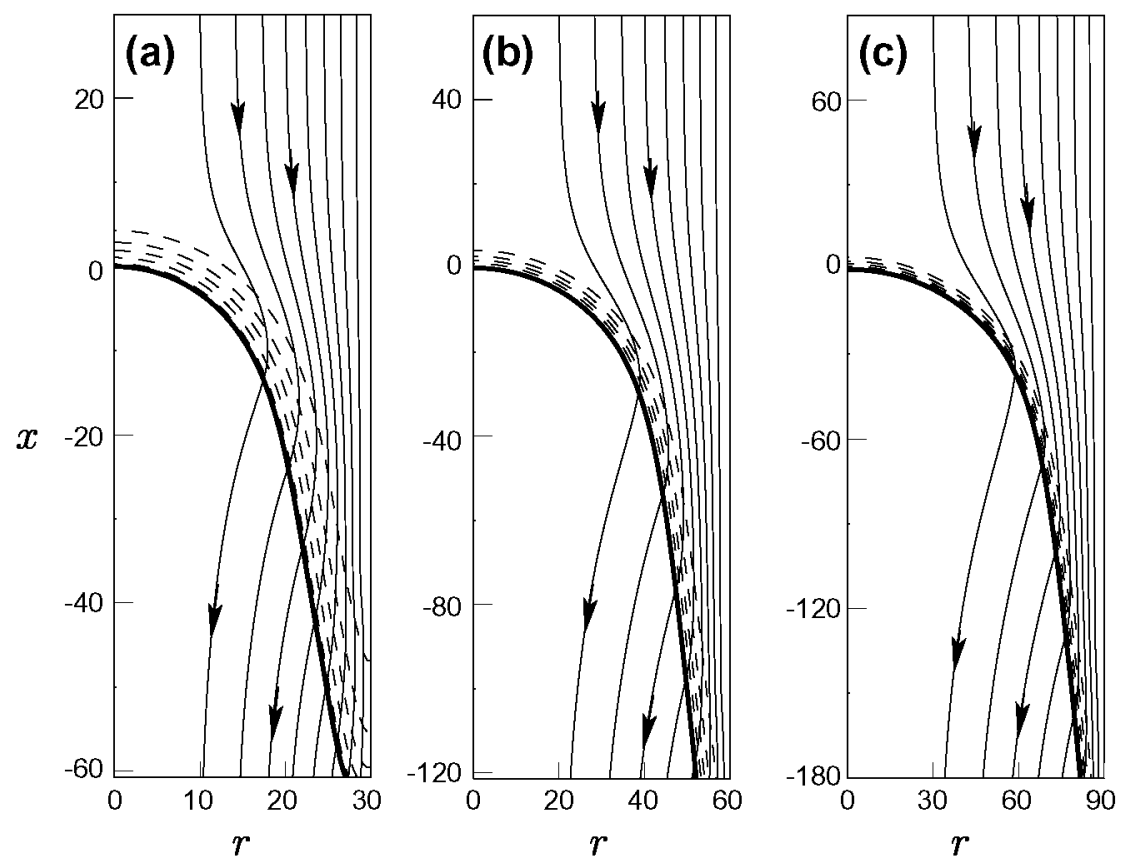

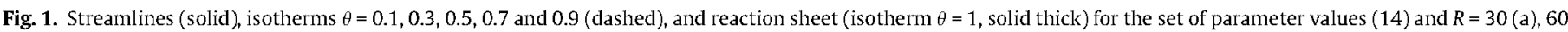
(b), and 90 (c).

bubble. This difference is small because the upward velocity of the flame, $U_{0}$, is fairly large compared to the velocity of the flame relative to the gas, of order unity in dimensionless variables. As a consequence, the tip of the flame is in a region of relatively small velocity (compared to $U_{0}$ ) and the rest of the flame is nearly aligned with the streamlines of the fresh gas flow.

The difference between the flow fields in Fig. 1 and those of rising bubbles is more pronounced in the burnt gas behind the flame. This gas moves downward in the three cases shown, while the light fluid in a bubble rising in a long tube recirculates and moves upward (relative to the interface) with a velocity which scales with $U_{D T}$ in a region around the axis of the tube. In the cases of Fig. 1, this upward motion is suppressed by the normal velocity of order $\gamma$ with which the burnt gas emerges from the flame. However, as will be seen in the following section, this situation may change and recirculation may appear in the burnt gas when the product $(G R)^{1 / 2}=\left(g R^{*}\right)^{1 / 2} / U_{L}$ [cf. (15)] is increased, either by increasing the radius $R^{*}$ of the tube, which makes the flow faster and nearer to that of a bubble, or by decreasing the velocity $U_{L}$ of the planar flame. The pressure variations due to the motion of the burnt gas, where $\rho=O(1 / \gamma)$, are of order $\rho|\boldsymbol{v}|^{2}=O\left[\max \left(\gamma, U_{0}^{2} / \gamma\right)\right]$, which are small compared to the dynamic pressure of the fresh gas flow, of $O\left(U_{0}^{2}\right)$.

\subsection{Conditions for stationary flame propagation}

The adiabatic flame temperature $T_{b}$ and the velocity of the planar flame $U_{L}$ decrease when the mass fraction of fuel in the mixture, $Y_{u}$, decreases. However, due to the exponential factor in (1), the variation of $U_{L}$ is much more pronounced than that of $T_{b}$, and thus the effect of $Y_{u}$ can be analyzed by changing $U_{L}$ only, with the other physical parameters kept constant. But, since $U_{L}$ and $\delta_{L}$ (which depends on $U_{L}$ ) are used to nondimensionalize the problem, changing $U_{L}$ amounts to changing the three dimensionless parameters $G, \Sigma$ and $R$ in (13). Here, to simplify comparison of different cases, the nondimensionalization will be modified using the values $U_{L_{\tau}}$ and $\delta_{L_{r}}$ introduced below (14), instead of the actual values of $U_{L}$ and $\delta_{L}$, to define dimensionless variables and parameters. The new nondimensionalization leaves (3)-(12) unchanged, except that a factor $U_{f}=U_{L} / U_{L_{r}}$ appears multiplying the last term of (9) at the reaction sheet, so that Eqs. (9) become

$$
\left.\begin{array}{l}
\theta=1, \quad[|H|]=0, \\
\left.\lambda(1) \frac{\partial \theta}{\partial n}\right|_{0^{-}}=\frac{\lambda(1)}{l e}\left[\left|\frac{\partial H}{\partial n}\right|\right]=U_{f} e^{H / 2},
\end{array}\right\}
$$

and that $U_{L_{r}}$ and $\delta_{L_{r}}$ should be understood in (13).

Figure 2, together with Fig. 1a and b, illustrates the evolution of the flow and the temperature distribution with $U_{f}$ when the
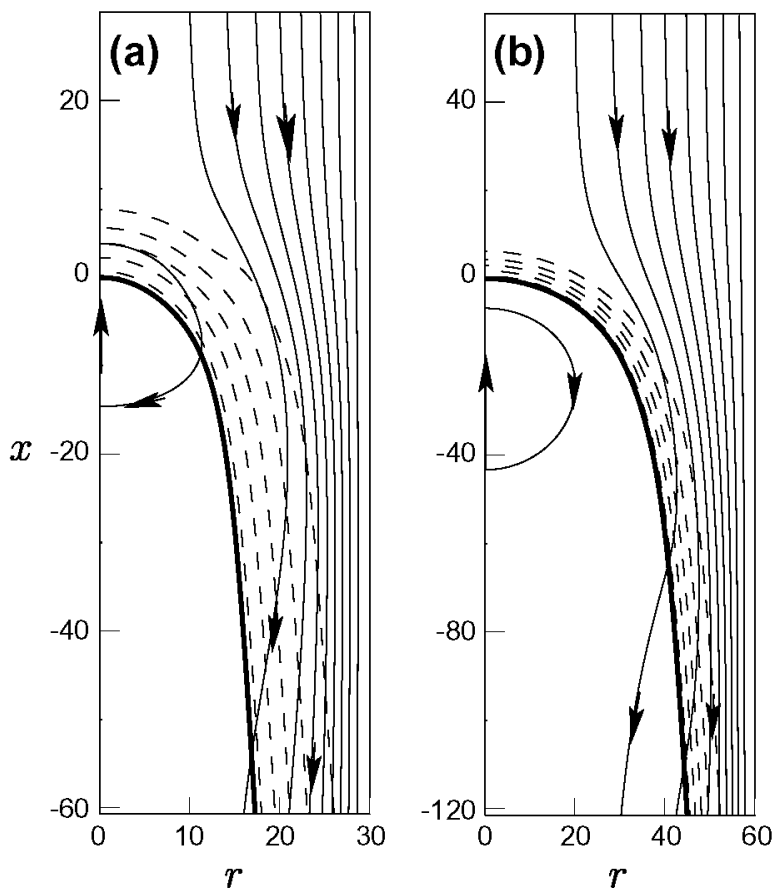

Fig. 2. Streamlines (solid), isotherms $\theta=0.1,0.3,0.5,0.7$ and 0.9 (dashed), and reaction sheet (isotherm $\theta=1$, solid thick) for the minimum $U_{f}$ at which a stationary solution is obtained, which is $U_{f}=0.48$ when $R=30$ (a) and $U_{f}=0.59$ when $R=60$ (b). Other parameters have the base values (14). 
modified dimensionless parameters take the values (14) and $R=30$ and 60 , respectively. ( $U_{f}=1$ in Fig. 1 with the modified nondimensionalization.) Comparison of Figs. 1 and 2 shows that the thickness of the flame increases when $U_{f}$ decreases, which is consistent with the decrease of $\partial \theta / \partial n$ in $\left(9^{\prime}\right)$. The velocity with which the burnt gas emerges from the flame, of order $\gamma U_{f}$, decreases with $U_{f}$, leading to the appearance of recirculation in the burnt gas when $U_{f}$ becomes smaller than a certain value which is about 0.59 for $R=30$ and about 0.68 for $R=60$. The size of the recirculation region increases when $U_{f}$ is further decreased, until a value of $U_{f}$ is reached at which the numerical computations cease to converge to a stationary solution. The minimum $U_{f}$ for which a stationary solution is obtained is 0.48 for $R=30$ and 0.59 for $R=60$.

Figure 3 shows the variation of some properties of the solution with $U_{f}$. The velocity of the flame $U_{0}$ depends weakly on $U_{f}$ [Fig. 3a]. It is nearly constant at a value close to $U_{D T}$ when $U_{f} / U_{D T}$ is small and increases slightly with $U_{f}$ when the velocity of the flame relative to the fresh gas ceases to be small compared to $U_{0}$.

The flame is stretched by the nonuniform flow where it is immersed. The strain rate of the fresh gas immediately ahead of the flame tip is $-\partial u / \partial x$, where $u$ is the vertical component of the gas velocity and the derivative is evaluated at the point on the tube axis where the temperature begins to increase. Figure $3 \mathrm{~b}$ shows that this strain rate increases when the radius of the tube or the velocity $U_{f}$ of the planar flame decrease. The first result has been explained [10-12] noticing that the strain rate in the fresh gas scales with $(G / R)^{1 / 2}$, as in the flow above a rising bubble [4]. The second result reflects the fact that the strain rate in the fresh gas increases on approaching the tip of the flame from above and tends to a maximum at the point where the velocity of the fresh gas tends to zero. Such stagnation point is not realized in the flow above a flame, contrary to the case of a rising bubble, but it is more closely approached when $U_{f}$ decreases and the flame tip is forced into a region of lower velocity. It may be noted that the solution of (7) with $l e=\Sigma=0$ and the homogeneous boundary conditions in (10)-(12) is $H=0$, so that the dimensionless burning rate of the flame, given by $\lambda(1) \partial \theta / \partial n$ at the upstream side of the reaction sheet, is a constant equal to the dimensionless burning rate of a planar unstretched flame with the same value of $U_{f}$; see $\left(9^{\prime}\right)$.

The structure of the flow slightly above the minimum $U_{f}$ depends on the radius of the tube. In narrow tubes $[R=30$, Fig. 2a], the tip of the reaction sheet gets into the recirculation region when $U_{f}$ decreases, so that, as for a planar flame in a stagnation point flow [7], reverse convection from the burnt gas helps keeping $\partial \theta / \partial n$ at the small value required by $\left(9^{\prime}\right)$. The flame, however, is far from planar in these conditions, as the ratio of its thickness $\left(\delta_{f}\right.$, defined as the distance from the isotherm $\theta=0.1$ to the reaction sheet) to the inverse of the mean curvature of the reaction sheet is not small; see solid curve of Fig. 3c. The curved flame fills the region of low velocity around its tip for the smallest values of $U_{f}$ for which a stationary solution has been found, which suggests that this region might not be able to lodge the flame for arbitrarily small values of $U_{f}$. In contrast, the flame in a wide tube $[R=60$, Fig. 2b] never enters the region of upward flow and is thin compared to its curvature radius at the tip, though the distance from the reaction sheet to the recirculation region becomes of the order of the flame thickness when the minimum $U_{f}$ is approached. (a)
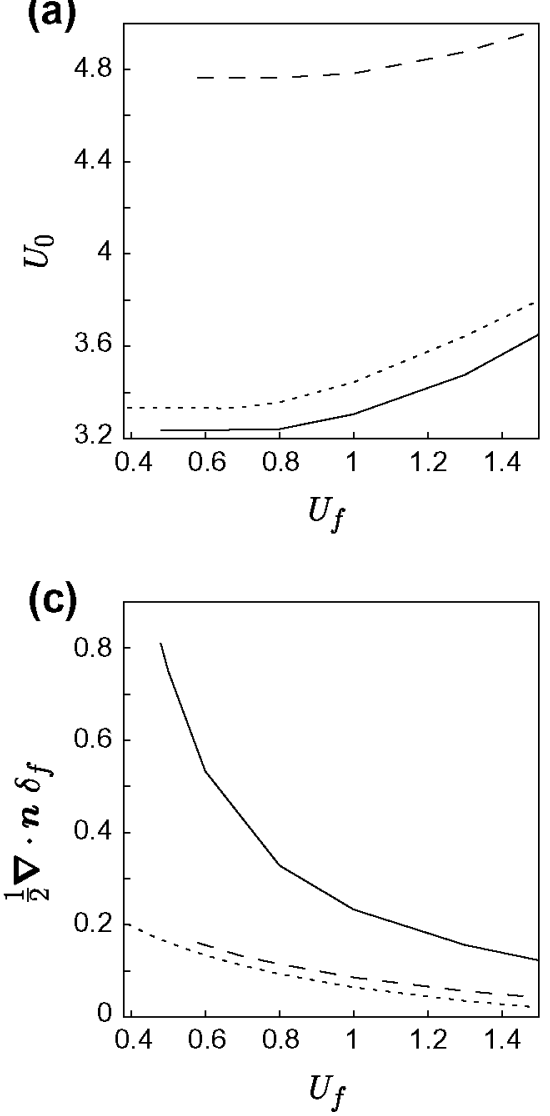

(b)
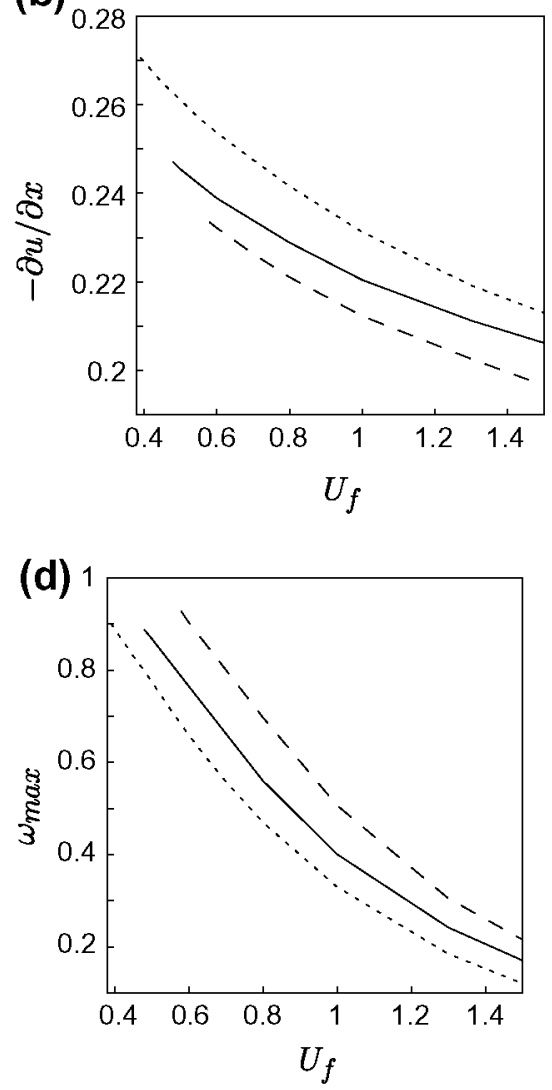

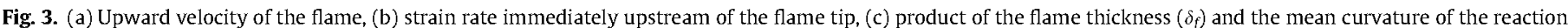

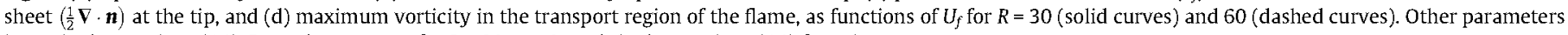
have the base values (14). Dotted curves are for $R=30, \kappa=0$, and the base values (14) for other parameters. 
Additional computations for constant values of the viscosity and conductivity of the gas $[\kappa=0$ in (8)] have been carried out to assess the effect of the temperature dependence of these transport properties. Some results are shown in Fig. 3 for $R=30$ (dotted curves). As can be seen, the results and their variation with $U_{f}$ are similar to those obtained with $\kappa=0.75$ (solid curves). The main difference occurs in Fig. 3c. The thickness of the flame, $\delta_{f}$, decreases when the variation of the conductivity with temperature is left out, and the curvature of the reaction sheet is then small compared to the inverse of this thickness for any value of $U_{f}$. The velocity and temperature fields (not shown) are similar to those of Figs. 1 and 2 , though the flame is somewhat thinner. Recirculation first appears for $U_{f} \approx 0.56$, and the minimum $U_{f}$ for which a stationary solution is found is about 0.39 , which is slightly smaller than the minimum $U_{f}$ for $\kappa=0.75$. The tip of the reaction sheet approaches the recirculation region when the minimum $U_{f}$ is approached but does not enter it. The limiting solution resembles the solution for $\kappa=0.75$ in a tube of larger radius.

A minimum $U_{f}$ (which amounts to a minimum concentration of fuel in the fresh gas) that increases with the radius of the tube, and below which no stationary upward propagation occurs, is in line the findings of many of the experiments reviewed in the introduction $[2,6,10,12,14]$. However, some of the explanations of this experimental result given in the literature are not applicable here. Thus, as has been already mentioned, the result has been attributed to the extension of the range of fuel concentrations where a flame can exist which is brought about by the combination of stretch and preferential diffusion with $L e<1$ [12], or to radiation losses from the burnt gas, which decrease the temperature of the flame at the tip and may cause extinction $[13,14]$. However, preferential diffusion and radiation losses have not been included in the computations presented in this section, which were carried out with $l e=\Sigma=0$. Moreover, in the framework of the single irreversible reaction with infinitely high activation energy that has been used, a planar unstretched flame exists in these conditions for any value of $U_{f}$, however small, and the burning rate and final combustion temperature of the flame are not affected by the stretch.

The failure of the computations to converge to a stationary solution below a minimum $U_{f}$ might be pointing to an instability of this stationary solution. Although the numerical method that has been used is not suitable to describe a time dependent flow, the manner it fails at the minimum $U_{f}$ suggests that the flow of the hot gas becomes pulsatory. Pending a rigorous stability analysis, it may be argued that a hydrodynamic instability is not unexpected in the complex flow that exists behind the flame at small values of $U_{f}$. In contrast to the irrotational bubble-like flow of the fresh gas ahead of the flame, the flow of the burnt gas is rotational due to the baroclinic production of vorticity in the whole transport region of the flame. Figure 4 illustrates the evolution of the vorticity field with $U_{f}$ and $R$, and Fig. $3 \mathrm{~d}$ shows the maximum vorticity in the flame as a function of $U_{f}$ for two tube radii. As can be seen, the vorticity accumulates at the flame, which is also a shear layer that induces an upward velocity around the axis of the tube. The thickness and strength of this layer increases when $U_{f}$ decreases because the thickness of the flame (where the vorticity is generated) increases, and the rate at which the vorticity is convected away by the gas crossing the flame decreases.

The vorticity layer is liable to become unstable when the vorticity maximum separates from the cold upper boundary (the cold fresh gas) which probably hinders the growth of the perturbations for larger $U_{f}$. The reaction sheet, where the temperature and the temperature gradient should have constant given values, also separates from the cold upper boundary, and its tip approaches the boundary of the recirculation region (and may even get into it) when $U_{f}$ decreases. This makes it possible for the vorticity-induced velocity perturbations to upset the conditions $\left(9^{\prime}\right)$ and elicit a tran-
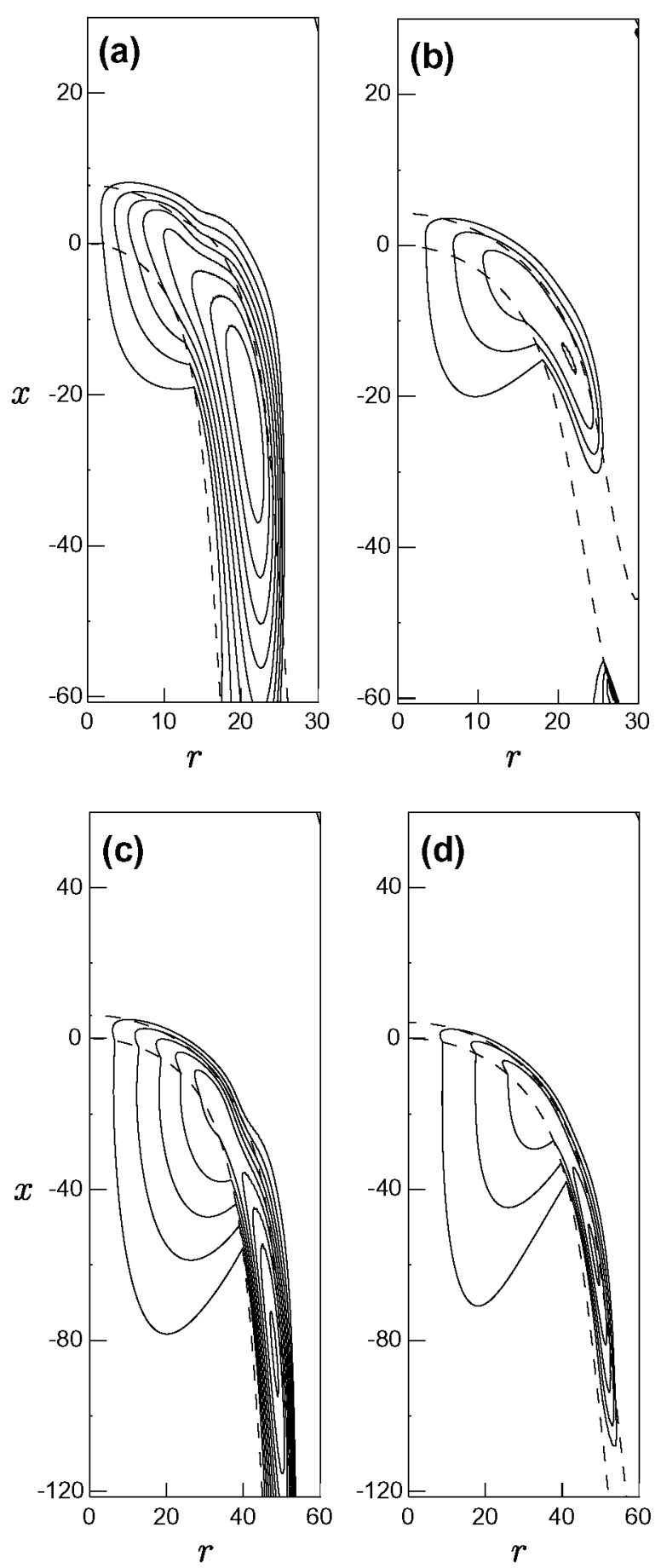

Fig. 4. Equispaced contours of the vorticity for $R=30$ with $U_{f}=0.48$ (a) and $U_{f}=1$ (b), and for $R=60$ with $U_{f}=0.59$ (c) and $U_{f}=1$ (d). The spacing of the vorticity contours is $\Delta \omega=0.1$ in all the panels. The dashed curves are the isotherms $\theta=0.1$ and 1 . Other parameters have the base values (14).

sient response of the flame. Figure $3 \mathrm{~d}$ also shows that, contrary to the strain rate, the maximum vorticity increases with the radius of the tube, which suggests that the flow in a wide tube may become unstable earlier than the flow in a narrow tube when $U_{f}$ is decreased.

The onset of this hypothesized instability may easily go unnoticed because, as was mentioned above, the pressure variations generated by the motion of the burnt gas are too small to significantly affect the flow of the fresh gas. The shape of the cold boundary and its upward velocity depend little on the details of the burnt 
gas flow, insofar as there is a flame that maintains the density difference on which the buoyancy force depends.

It may also be noted that the stationary solutions discussed in this section might not be always realized in tubes of finite length, even if these solutions are stable, because the time it takes for a flow with a large recirculation region to become stationary is of the order of the diffusion time in this region, which may be longer than the transit time of the flame along the length of the tube.

\subsection{Effects of the Lewis number and the radiation}

The excess of enthalpy $H$ defined in (2) ceases to be null when $l e \neq 0$. Its value at the reaction sheet, where $Y=0$, is the difference between the final combustion temperature and the adiabatic flame temperature scaled with $\beta^{-\mathbf{1}}$. In agreement with well-known results for planar stretched flames [7], the numerical results for $l e \neq 0$ and $\Sigma=0$ show that $H$ is positive at the reaction sheet when $l e<0$ and negative when $l e>0$, and that the absolute value of $H$ is maximum at the tip of the flame, where the strain rate is maximum. These results are also in agreement with the numerical computations of Shoshin et al. [12] for lean methane-air flames in flammability tubes. Figure 5a shows the value of $H$ at the tip as a function of $U_{f}$ for different values of le. The dimensionless burning
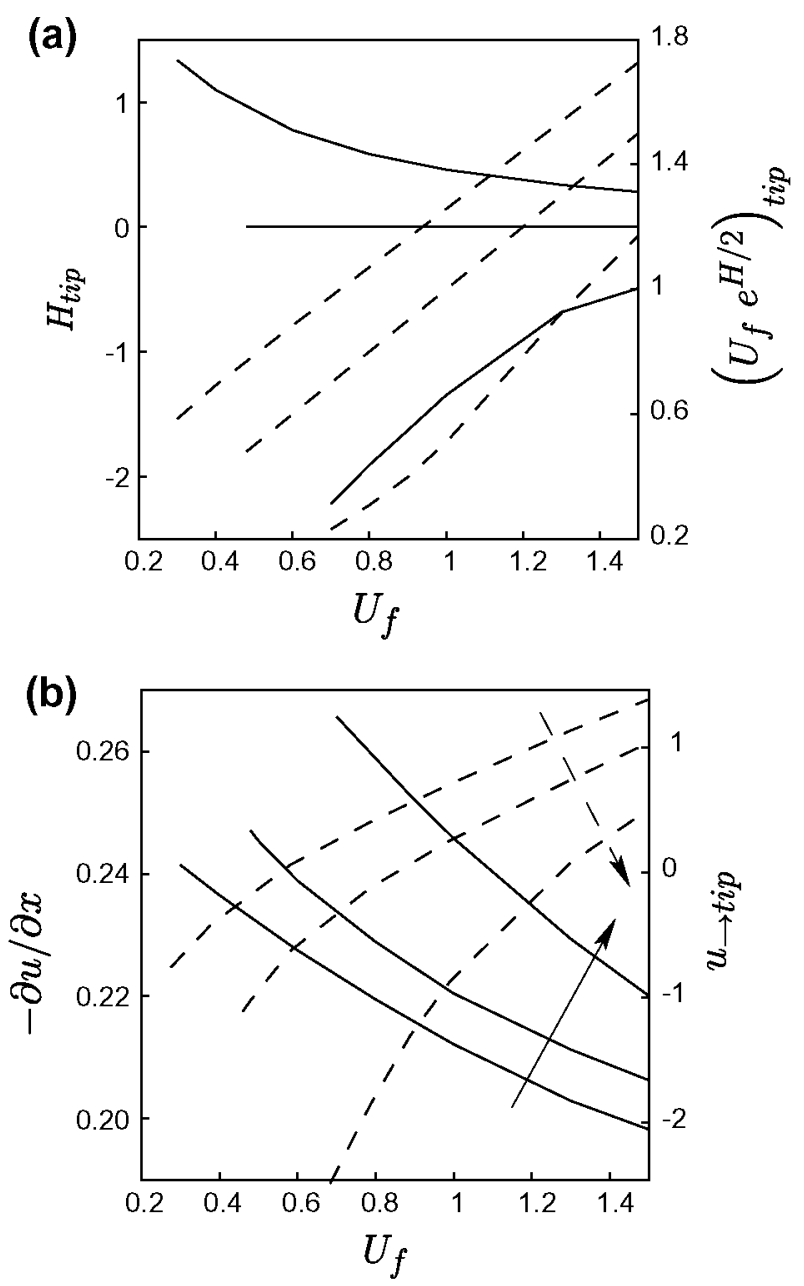

Fig. 5. (a) Excess of enthalpy $H$ (solid) and dimensionless burning rate $U_{f} \exp (H / 2)$ (dashed, right-hand side scale) at the tip of the reaction sheet as functions of $U_{f}$ for $l e=-2,0$ and 2 , increasing from top to bottom. (b) Strain rate immediately upstream of the flame tip (solid) and velocity of the fresh gas extrapolated to the reaction sheet (dashed, right-hand side scale) as functions of $U_{f}$ for $l e=-2,0$ and 2 , increasing as indicated by the arrows. Here $R=30$ and other parameters have the base values (14). rate at the tip $\left[U_{f} \exp (H / 2)\right.$; dashed curves in Fig. $\left.5 \mathrm{a}\right]$ is still an increasing function of $U_{f}$ but it decreases when le increases. The strain rate of the cold gas flow immediately upstream of the flame tip increases with $l e$, and the velocity of the fresh gas extrapolated to the reaction sheet along the axis of the tube decreases with le; see Fig. 5b. These results imply that, for a given $U_{f}$, the increase of the final combustion temperature that occurs around the tip when $l e<0$ increases the normal velocity of the flame relative to the cold gas and allows the flame to stand farther from the virtual stagnation point of the cold gas than when $l e=0$. The velocity with which the burnt gas emerges from the flame also increases with $-l e$, and this postpones the appearance of recirculation and the ultimate failure of the numerical computations to converge to a stationary solution to smaller values of $U_{f}$ than when $l e=0$. These trends reverse, and the minimum $U_{f}$ increases, when le takes positive values.

Radiation losses cause a defect of enthalpy by acting on both sides of the reaction sheet. This is contrary to the effective source of $H$ due to a $l e \neq 0$, which is confined to the transport region and the reaction sheet. Radiation, as well as a $l e>0$, may cause extinction of a stretched flame [7]. However, in the cases that have been computed, the effect of increasing radiation losses is qualitatively similar to the effect of decreasing $U_{f}$ in the absence
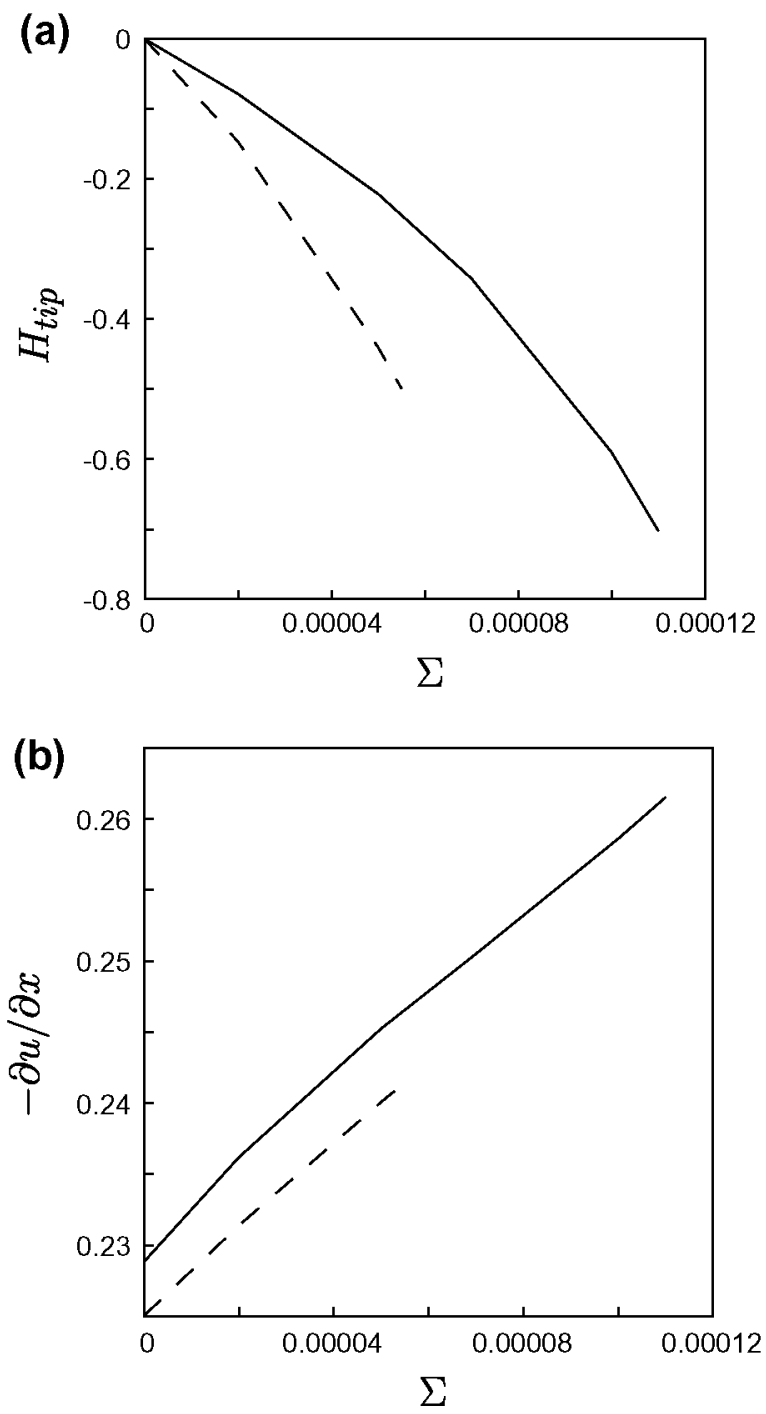

Fig. 6. Excess of enthalpy $H($ a $)$ and strain rate (b) at the tip of the flame as functions of $\Sigma$ [defined in Eq. (13)] for $U_{f}=0.8$ and $R=30$ (solid) and 60 (dashed). Other parameters have the base values (14). 
of radiation. When $\Sigma$ increases, (i) the final combustion temperature decreases, mainly at the tip of the flame [Fig. 6a], in agreement with the experimental results of Shoshin and Jarosinski [13]; (ii) the strain rate ahead of the flame increases [Fig. 6b]; and (iii) the downward velocity of the burnt gas decreases, until recirculation appears at a certain value of $\Sigma$ and the computations cease to converge to a stationary solution at a value of $\Sigma$ slightly larger than this. The maximum $\Sigma$ at which a stationary solution is found (end points of the curves in Fig. 6) decreases when the radius of the tube increases.

For a given $\Sigma$, the total heat loss is proportional to the volume of slowly moving hot gas; i.e., to $R^{3}$, whereas the area of the flame, where the heat is released, is proportional to $R^{2}$. Therefore the decrease of temperature at the tip $\left[-H_{\text {tip }}\right.$ in Fig. 6a] is more pronounced for large $R$ than for small $R$.

\section{Conclusions}

Numerical computations of the flow around a curved flame propagating stationarily upward in a vertical tube filled with a lean fresh mixture have been carried out using a single irreversible Arrhenius reaction model with infinitely high activation energy. The computed velocity of the flame is close to the velocity of an open bubble ascending in a tube of the same radius, insofar as this velocity is large compared to the velocity of the flame relative to the fresh gas and the Lewis number of the fuel is not far from unity (reduced Lewis number le defined in (13) of order unity). The burning rate of a planar flame propagating in the fresh mixture is used as a measure of the mass fraction of fuel in the mixture. In the absence of radiation losses and preferential diffusion $(l e=0)$, stationary numerical solutions of this purely hydrodynamic model have been obtained only above a certain value of the burning rate which increases with the radius of the tube.

Buoyancy forces dominate the flow of the fresh gas and determine the shape and velocity of the flame, but the flow of the hot gas is more complex than the flow of the light fluid in an ascending bubble. Vorticity is generated by baroclinic couples in the transport region of the flame and is convected by the gas that crosses the flame. This vorticity induces an upward velocity in the burnt gas which is opposed by the downward velocity with which this gas emerges from the flame. When the burning rate of the flame is decreased by decreasing the mass fraction of fuel, the first of these velocities dominates, leading to a recirculation region in the burnt gas, and the thickness of the flame and the maximum vorticity increase, which may promote instability of the hot gas flow. The pattern of this flow in the vicinity of the numerical limit of convergence (minimum burning rate at which a stationary solution has been found) depends on the radius of the tube. In narrow tubes, the reaction sheet gets into the recirculation region and the thickness of the flame becomes of the order of its curvature radius when the numerical limit of convergence is approached. In wide tubes, however, the flame is relatively thin compared to its curvature radius and stays at a distance from the recirculation region of the order of its thickness. It is argued that the strong flame stretch due to the flow and the curvature of the flame tip in narrow tubes, and the instability of the hot gas flow, may be responsible for the minimum burning rate found numerically.

A le $<0$ increases the final combustion temperature at the tip of the flame and extends the converged stationary solution to leaner mixtures. Radiation losses or a $l e>0$ have the opposite effect and increase the minimum fuel mass fraction for which a stationary solution can be computed.

\section{Acknowledgments}

This work was supported through projects HYSYCOMB, S2009/ENE-1597 (Comunidad de Madrid) and CSD2010-00010 (Ministerio de Ciencia e Innovación).

\section{References}

[1] H.F. Coward, G.W. Jones, US Bureau of Mines Bull. 503 (1952).

[2] A. Levy, Proc. R. Soc. London, A 283 (1965) 134.

[3] J. Jarosinski, R.A. Strehlow, A. Azarbarzin, Proc. Combust. Inst. 19 (1982) 1549

[4] R.M. Davies, G.I. Taylor, Proc. R. Soc. London, A 200 (1950) 375.

[5] Y.M. Shtemler, G.I. Sivashinsky, Combust. Sci. Technol. 102 (1994) 81

[6] V.S. Babkin, V.V. Zamashchikov, A.M. Badalyan, V.N. Krivulin, E.A. Kudryavtsev, A.N. Baraton, Fizika Goreniya i Vzryva 18 (1982) 4

[7] F.A. Williams, Combustion Theory, 2nd ed., Benjamin Cummings, Menlo Park, CA, 1985 (Chapters 5 and 10).

[8] B. Lewis, G. von Elbe, Combustion, Flames and Explosions of Gases, Academic Press, New York, 1961 (Chapter 5).

[9] M. Hertzberg, Bureau of Mines Report \#8127 (1976).

[10] E. von Lavante, R.A. Strehlow, Combust. Flame 49 (1983) 123

[11] J. Buckmaster, D. Mikolaitis, Combust. Flame 45 (1982) 109.

[12] Y.L. Shoshin, L. Tecce, J. Jarosinski, Combust. Sci. Technol. 180 (2008) 1812.

[13] Y. L Shoshin, J. Jarosinski, Proc. Combust. Inst. 32 (2009) 1043.

[14] Y.L. Shoshin, G. Gorecki, J. Jarosinski, T. Fodemski, Combust. Flame 157 (2010) 884.

[15] D.B. Spalding, Proc. R. Soc. London, A 240 (1957) 83.

[16] F.J. Higuera, Combust. Flame 157 (2010) 1586. 